\title{
Epidemiological study of Guillain-Barré syndrome in south east England
}

\author{
J H Rees, R D Thompson, N C Smeeton, R A C Hughes
}

\begin{abstract}
Objectives-To determine the incidence, treatment, and outcome of Guillain-Barré syndrome in south east England.

Methods-Patients presenting with confirmed Guillain-Barré syndrome between 1 July 1993 and 30 June 1994 were recruited via a voluntary reporting scheme coordinated by the British Neurological Surveillance Unit, hospital activity data collected from acute admitting hospitals within the South East and South West Thames Regional Health Authorities, death certificates, and a contemporary research study of Guillain-Barré syndrome and Campylobacter jejuni infection. All patients were followed up for one year to determine outcome.
\end{abstract}

Results-Seventy nine patients were recruited, 35 (44\%) male, 44 (56\%) female, including three children (two boys, one girl). The crude ( $95 \%$ confidence interval (95\% CI)) annual incidence was 1.2 (0.91.4) cases $/ 100000$ population and 1.5 (1.31.8)/100 000 when adjusted for undetected cases. Twenty (25\%) patients required ventilation for an average (SD) of 42 (64) days. Thirty six $(46 \%)$ patients received intravenous human immunoglobulin, five $(6 \%)$ received plasma exchange, 11 (14\%) both treatments, three (4\%) steroids, and $25(32 \%)$ no immunomodulatory treatment. One year later, six patients $(8 \%)$ had died, all of whom were older than 60 , three (4\%) remained bedbound or ventilator dependent, seven ( $9 \%$ ) were unable to walk unaided, $14(17 \%)$ were unable to run, and $49(62 \%)$ had made a complete or almost complete recovery. Increasing age was significantly associated with a poorer outcome at one year.

Conclusions-Despite the frequent use of modern immunomodulatory treatments Guillain-Barré syndrome still carries considerable morbidity and mortality. (F Neurol Neurosurg Psychiatry 1998;64:74-77) Health Medicine, UMDS Guy's Hospital, St Thomas' Street, London, UK

N C Smeeton

Correspondence to: Professor R A C Hughes, Department of Neurology, UMDS Guy's Hospital, St Thomas' Street, London SE1 9RT, UK.

Received 25 March 1997 and in final revised form 1 September 1997 Accepted 17 September 1997

Guillain-Barré syndrome is the commonest cause of acute neuromuscular paralysis in most countries. Incidence figures vary from 0.4 to $4 / 100000$ population/year, ${ }^{1}$ the higher figures being reported from detailed studies of small populations. Plasma exchange has been shown to shorten the duration of ventilation and inability to walk, with significant savings in healthcare costs. ${ }^{2}$ Intravenous human immu- noglobulin (IgG) has equivalent clinical effects. ${ }^{45}$ The effects of these treatments on long term prognosis, however, is not known. We have ascertained the incidence of GuillainBarré syndrome in a south east England population in 1993-4 and compared the prognosis with that published from a similar population in $1983-4 .^{6}$

\section{Methods}

Patients of all ages presenting with the onset of Guillain-Barré syndrome between 1 July 1993 and 30 June 1994 and residing in south east England (comprising south east and south west London, and Surrey, Kent, and Sussex counties with a mid-1993 census population of 6716 917) were recruited in four ways:

(1) A voluntary reporting scheme coordinated by the British Neurological Surveillance Unit (BNSU) $(n=23)$. All consultant neurologists and neurophysiologists within the South Thames and immediately surrounding Health Authority regions were invited to report new cases of Guillain-Barré syndrome via a monthly postal questionnaire.

(2) Hospital activity analysis $(n=68)$. In August 1994, all acute admitting hospitals within the South East and South West Thames Regional Health Authorities (RHAs) provided a list of patients with a primary diagnosis of Guillain-Barré syndrome (code 357.0 in the International Classification of Diseases, ninth revision (ICD-9)). We also obtained hospital activity analysis data from the South Thames regional headquarters. These data would not have included patients admitted into hospitals in neighbouring regions but residing within the South Thames regions. Such "cross boundary" referrals should however have been included in data collected from 1, 3, and 4. Non-residents and readmissions were excluded.

(3) A contemporary research database $(n=22)$. We actively sought patients with Guillain-Barré syndrome as part of a synchronous study investigating the relation between previous Campylobacter jejuni infection and Guillain-Barré syndrome ${ }^{7}$ by personal approaches to consultant neurologists, physicians, and their admitting teams in south east England and surrounding regions.

(4) Death certificates $(n=5)$. We obtained a list of patients who died from Guillain-Barré syndrome from the Office for Population and Census Studies (OPCS). Further details were then obtained from the responsible consultant.

The total number of 118 exceeds the final 79 as some patients' details were collected from more than one source. To estimate the number of "missed" cases of Guillain Barré syndrome 
in the region, capture-recapture analysis ${ }^{8}$ was performed on the four sources of cases using the statistical package GLIM. ${ }^{9}$ A $95 \%$ confidence interval $(95 \% \mathrm{CI})$ was calculated for the estimate using the goodness of fit method of Regal and Hook, ${ }^{10}$ chosen because many of the subgroups were very small.

We checked the diagnosis from the contemporary records (all groups) and followed up patients for one year personally (group 3) or by telephone consultation with the family doctor (groups 1 and 2). Persistent disability was graded using a scale from a contemporary study $^{7}(0=$ healthy: no signs or symptoms due to Guillain-Barré syndrome, $1=$ minor symptoms or signs and capable of running; $2=$ able to walk $5 \mathrm{~m}$ across an open space without assistance, walking frame, or stick but unable to run; $3=$ able to walk $5 \mathrm{~m}$ across an open space with the help of one person and waist level walking frame or sticks; $4=$ chairbound or bedbound: unable to walk as in $3 ; 5=$ requiring assisted ventilation for at least part of day or night; $6=$ dead).

A clinical diagnosis was accepted as Guillain-Barré syndrome if that diagnosis had been made by a consultant neurologist or by JHR, and the case fulfilled the criteria of Asbury and Cornblath (appendix). ${ }^{11}$ Case notes or discharge summaries were scrutinised for those patients recruited into group 2 . Patients were excluded if the diagnosis was not certain, if they had chronic inflammatory demyelinating polyradiculoneuropathy, or if no data were returned despite an initial request, two written reminders, and two telephone reminders. Two patients with Miller Fisher syndrome (ophthalmoplegia, ataxia, and areflexia $)^{12}$ were included as both had bulbar weakness and one had mild proximal limb weakness.

We compared the outcome with that of 100 patients recruited in 1983-4 from a somewhat larger area in south east England by personal approaches to consultant neurologists as in method 3. ${ }^{6}$ We also compared the outcome between patients who had been admitted or transferred to neurology centres (Guy's Hospital, St Thomas' Hospital, Atkinson Morley Hospital, Royal Surrey County Hospital, Hurstwood Park Neurology Centre, King's College Hospital, and Brook General Hospital) with those who had remained in district general hospitals throughout the duration of their admission using a $\chi^{2}$ test for linear trend comparing one year disability grades between neurology centres and district general hospitals.

We also performed logistic regression analysis to determine the effect of a number of variables on one year outcome, dichotomised to good (disability grade 0 or 1 ) or bad (disability grade $\geqslant 2$ ) outcome. The variables tested were age, sex, previous diarrhoeal illness, treatment with IgG or plasma exchange, mechanical ventilation, transfer to or admission to a neurology centre and comorbidity (defined as the need for concurrent regular medications before developing Guillain Barré syndrome).

The study had been approved by our ethics committee.

\section{Results}

PATIENTS

We identified 124 possible patients by all the above methods but excluded 45 because of incorrect diagnosis or coding $(n=32)$ or insufficient data $(n=13)$. We confirmed the diagnosis in 79 patients, 43 from the South East Thames Regional Health Authority and 36 from the South West Thames Regional Health Authority. Thirty five were men or boys and 44 women or girls $(\mathrm{M} / \mathrm{F}$ ratio $0.8 / 1)$. The mean (SD) age was 47.7 (19.5) years and the range was 5 to 85 years. Three children (two boys and one girl) were included. All but two patients were white, one being Indian and one black-Caribbean (according to the classification of ethnic origin defined by the OPCS).

The capture-recapture analysis led to an estimate of 19 undetected cases (95\% CI 7-41). This implies an estimate for the total number of cases of 98 (95\% CI 86-120).

\section{INCIDENCE}

The crude annual incidence rate $(95 \% \mathrm{CI})$ of Guillain-Barré syndrome was $1.2 / 100000$ (0.9-1.4). When adjusted for undetected cases this becomes 1.5/100 000 (1.3-1.8). Age adjusted incidence rates were roughly similar in females $(1.3(0.9-1.5))$ and males (1.1 (0.71.4)). The age-incidence distribution was somewhat bimodal with peaks at 15-24 years and 65-74 years. The incidence rates were identical in eastern and western parts of the region.

\section{HOSPITAL TREATMENT}

The mean (SD) duration of stay in hospital was 50 (53) days and the median (range) 31 (one to more than 365$)$ days. Twenty (25\%) patients were ventilated for a mean (SD) of 23 (22) and a median of 19 days (range four to more than 365 days). Treatment with either intravenous human immunoglobulin (IgG), plasma exchange, or both was given in $52(66 \%)$ cases and started within a mean (SD) of 3.9 (4.3) days and a median (range) of $2(0-22)$ days from admission. Thirty six patients $(46 \%)$ received IgG, five (6\%)plasma exchange, 11 $(14 \%)$ both treatments, and $25(32 \%)$ no treatment at all. Three $(4 \%)$ patients received corticosteroids, one also being treated with IgG.

Reasons given for not using plasma exchange or IgG were mild disease $(n=11)$, not recorded (nine), difficulty with diagnosis (two), patient death (one), patient refusal (one) and absence of plasma exchange facilities (IgG not suggested) (one).

\section{COMPLICATIONS OF TREATMENT}

Complications of treatment occurred in two of 36 patients $(6 \%)$ given IgG (acute on chronic renal failure and meningitis), two of 16 (13\%) patients given IgG and plasma exchange, both attributable to plasma exchange (hypocalcaemia and line infection with neck abscess formation), and none of five given plasma exchange alone. 
Table 1 Causes of death

\begin{tabular}{|c|c|c|c|c|c|}
\hline Age/sex & Cause of death & Day of death & $\begin{array}{l}\text { Antecedent } \\
\text { event }\end{array}$ & Ventilation & Treatment \\
\hline $85, \mathrm{~F}$ & $\begin{array}{l}\text { Respiratory and heart } \\
\text { failure }\end{array}$ & 2 & Nil & No & Nil \\
\hline $82, \mathrm{~F}^{\star}$ & Respiratory arrest & 15 & $\begin{array}{l}\text { Diarrhoea, } \\
\text { UTI }\end{array}$ & Yes & Nil \\
\hline $64, M$ & $\begin{array}{l}\text { Multisystem failure } \\
\text { Bronchopneumonia }\end{array}$ & 24 & Prostatectomy & Yes & $\mathrm{PE} / \mathrm{IgG}$ \\
\hline $67, \mathrm{~F}$ & Pneumonia & 29 & URTI & Yes & $\mathrm{PE} / \mathrm{IgG}$ \\
\hline $79, \mathrm{~F}$ & Pulmonary embolus & 54 & Nil & No & $\mathrm{IgG}$ \\
\hline $61, M$ & Unknown & 240 & Nil & No & $\mathrm{PE} / \mathrm{IgG}$ \\
\hline
\end{tabular}

$\star$ Relapsed after ventilation discontinued.

$\mathrm{PE}=$ plasma exchange $; \mathrm{IgG}=$ intravenous immunoglobulin .

Table 2 Logistic regression of variables leading to a poor outcome

\begin{tabular}{llll}
\hline Variable & Odds ratio & $95 \%$ CI & p Value \\
\hline Age & 1.06 & $1.02-1.10$ & 0.002 \\
Sex & 0.68 & $0.21-2.25$ & 0.53 \\
Previous diarrhoea & 3.33 & $0.70-15.83$ & 0.13 \\
Treatment with IgG & 1.90 & $0.57-6.31$ & 0.29 \\
Treatment with PE & 0.99 & $0.19-5.11$ & 0.99 \\
Ventilation & 1.82 & $0.45-7.31$ & 0.40 \\
Site (neurology centre or district general hospital) & 0.32 & $0.09-1.10$ & 0.07 \\
Comorbidity & 3.18 & $0.71-14.21$ & 0.12 \\
\hline
\end{tabular}

ONE YEAR OUTCOME

One year later, 49 patients (62\%) had made a complete functional recovery having no or minimal residual symptoms. However, 30 $(38 \%)$ patients had made a poor functional recovery, $14(18 \%)$ being unable to run, seven $(9 \%)$ being unable to walk unaided, three $(4 \%)$ being bedbound or ventilated, and six ( $8 \%$ ) having died (table 1). On a logistic regression analysis of multiple prognostic factors (age, sex, previous diarrhoeal illness, treatment with IgG or plasma exchange, ventilation, site (neurology centre or district general hospital), comorbidity), only increasing age was significantly associated with a poor outcome as defined by achieving a disability grade $\geqslant$ two $(\mathrm{p}=0.002$; odds ratio 1.06 for each incremental year; 95\% CI 1.02-1.10). A $\chi^{2}$ analysis for linear trend showed that patients treated in neurology centres fared better than those in district general hospitals $(p=0.02)$ but this did not reach $5 \%$ significance $(\mathrm{p}=0.07$; odds ratio 0.32 ; $95 \%$ CI $0.09-1.10$ ) on logistic regression analysis (table 2). When the effect of age was analysed in relation to the effect of site, the $p$ value became less significant $(p=0.09$; odds ratio $0.40 ; 95 \%$ CI $0.14-1.15)$. Conversely when the patients were divided into two groups - $<60$ years and $\geqslant 60$ years-the effect of site on outcome just achieved a significance level of $5 \%(\mathrm{p}=0.05$; odds ratio $0.35 ; 95 \%$ CI $0.12-1.00)$

\section{Discussion}

Previous population-based studies of incidence of Guillain-Barré syndrome ${ }^{13-20}$ did not consider the uptake of IgG and plasma exchange treatment and so were not able to examine outcome in treated patients. To overcome the problem of incomplete case ascertainment, we used four sources of information to recruit as many patients as possible and capturerecapture methods to estimate the total number of cases. The uptake of treatment, proportion of patients ventilated, duration of admission to hospital, and mortality were simi- lar to those of a group of 140 patients collected over a longer period and from a larger area as part of a separate study. We had insufficient data on $13(16 \%)$ possible patients and may have missed patients who were not diagnosed or not admitted to hospital. Despite these reservations, our incidence figure of 1.2/100 000 or 1.5/100 000 with capture-recapture analysis is similar to that published from Oxfordshire ${ }^{18}$ and was identical in the eastern and western parts of our region.

The few patients $(n=23)$ recruited via the BNSU scheme suggest that either patients with Guillain Barré syndrome are never seen by a neurologist or that voluntary reporting schemes are susceptible to underreporting. Analysis of hospital activity data confirmed that there were at least 45 more cases which had not been reported via the BNSU card scheme, indicating that hospital discharge data are more sensitive than passive surveillance in detecting cases. ${ }^{21}$ We noted many errors and duplications among the coding data, necessitating a careful review of all case notes or discharge summaries before accepting a diagnosis of Guillain-Barré syndrome or Miller Fisher syndrome

We were pleased to discover that plasma exchange or IgG had been given in a timely fashion (median time to instituting treatment only two days) in most cases when it seemed appropriate. The longest delay in starting treatment, 22 days, occurred in an unusual case involving transfer from abroad and uncertainty about the diagnosis. Because of its greater ease of administration, the preferred treatment was IgG rather than plasma exchange, as IgG needs neither the complex machinery nor the specially trained personnel required for plasma exchange. Plasma exchange combined with IgG has not shown a clinically significant advantage. ${ }^{5}$

To compare this cohort of patients treated when deemed appropriate with IgG or plasma exchange, we have made use of the cohort of 100 patients collected by Winer et al ${ }^{6}$ in 1983-4 when only $10(10 \%)$ received plasma exchange and none received IgG. In our study $25 \%$ of patients needed ventilation compared with $23 \%$ in Winer's study, $8 \%$ died compared with $13 \%, 30 \%$ had a poor outcome (defined as being unable to run, walk unaided, being chairbound or bedbound, or requiring ventilation) compared with $20 \%$, and only $62 \%$ made a good recovery compared with $67 \%$. Plasma exchange has been calculated to save so much intensive care unit and hospital time that it reduces healthcare costs ${ }^{22}$ but it has not been shown to reduce the percentage with severe persistent disability. The costs and benefits of IgG are about the same as plasma exchange in most institutions. Unfortunately our data show that these treatments do not prevent death and disability. Demonstration of moderate reductions in these outcomes would require much larger randomised trials than have been performed. Such trials would probably not be ethical in view of the established short term benefits of plasma exchange and similar efficacy of IgG. ${ }^{235}$ 
We have identified a trend towards improved outcome of patients treated for all or part of their inpatient stay at neurology centres. The effect seems to be independent of other variables which may have influenced prognosis and, in particular, is not confounded by considerations of age-that is, a trend for younger patients to be referred up to neurology centres. Further studies are necessary to determine whether this is a real effect of specialist care, a selection bias, or a type 1 error.

Further efforts are needed to improve delivery of acute care, to avoid deaths and complications, and to identify more effective treatments particularly for elderly patients, who fare significantly worse than younger patients.

We gratefully acknowledge the British Neurological Surveillance Unit and all the doctors who provided information on their patients. We thank Mr Jonathan Elf, Havering Hospitals NHS Trust, for providing age adjusted incidence figures.

JHR received a Medical Research Council Training Award.

Conflict of interest: Professor Hughes' department has received funding from Sandoz to undertake a trial comparing intravenous IgG with plasma exchange in Guillain Barré syndrome.

\section{Appendix: Diagnostic criteria for Guillain-Barré syndrome (Asbury and Cornblath $^{11}$ )}

I Features required for diagnosis

A Progressive motor weakness of more than one limb.

B Areflexia

II Features strongly supportive of the diagnosis

A1 Progression within four weeks

2 Relative symmetry

3 Mild sensory symptoms or signs

4 Cranial nerve involvement

5 Recovery within four weeks of progression stopping

6 Autonomic dysfunction

7 Absence of fever at onset

B1 Raised CSF protein

2 CSF mononuclear leucocyte count less than $10 / \mathrm{mm}^{3}$

C Electrodiagnostic features strongly supportive of the diagnosis (nerve conduction slowing or block)

III Features casting doubt on the diagnosis

1 Pronounced persistent asymmetry of weakness

2 Persistent bladder or bowel dysfunction

3 Bladder or bowel dysfunction at onset

4 More than 50 mononuclear leucocytes/ $\mathrm{mm}^{3}$

5 Presence of polymorphonuclear leucocytes in CSF
6 Sharp sensory level

IV Features that rule out the diagnosis

1 Current history of hexacarbon misuse

2 Abnormal porphyrin metabolism

3 Recent diphtheritic infection

4 Features clinically consistent with lead neuropathy

5 Purely sensory syndrome

6 Definite diagnosis of poliomyelitis, botu-

lism, hysterical paralysis, or toxic neuropathy.

1 Alter M. The epidemiology of Guillain-Barré syndrome. Ann Neurol 1990;27(suppl):S7-12.

2 Guillain-Barré Syndrome Study Group. Plasmapheresis and acute Guillain-Barré syndrome. Neurology 1985;35:1096104.

3 French Cooperative group in plasma exchange in GuillainBarré syndrome. Efficiency of plasma exchange in GuillainBarré syndrome: role of replacement fluids. Ann Neurol 1987;22:753-61.

4 Van der Meche FGA, Schmitz PIM, Dutch Guillain-Barré Study Group. A randomised trial comparing intravenous immune globulin and plasma exchange in Guillain-Barré syndrome. N Engl f Med 1992;326:1123-9.

5 The Plasma Exchange/Sandoglobulin Guillain-Barré syndrome Trial Group. Randomised trial of plasma exchange, drome Trial Group. Randomised trial of plasma exchange, intravenous immunoglobulin, and combined treatmen

6 Winer JB, Hughes RAC, Osmond C. A prospective study of acute idiopathic neuropathy. I. Clinical features and their prognostic value. F Neurol Neurosurg Psychiatry 1988;51: 605-12.

7 Rees JH, Soudain SE, Gregson NA, et al. Campylobacter jejuni infection and Guillain-Barré syndrome. $N$ Engl f Med 1995;333:1374-80.

8 Bishop YMM, Fienberg SE, Holland PW. Discrete multivariate analysis: theory and practice. Cambridge, Massachusetts: MIT Press, 1975.

9 Payne CD, ed. The GLIM system: release 3.77. Oxford: NAG, 1986

10 Regal RR, Hook EB. Goodness-of-fit confidence intervals for estimates of the size of a closed population. Stat Med 1984;3:287-91.

11 Asbury AK, Cornblath DR. Assessment of current diagnostic criteria for Guillain-Barré syndrome. Ann Neurol 1990; 27(suppl):S21-4.

12 Miller Fisher C. An unusual variant of acute idiopathic polyneuritis (syndrome of ophthalmoplegia, ataxia and areflexia). $N$ Engl f Med 1956;255:57-65.

13 Jiang GX, de Pedro-Cuesta J, Fredrikson S. Guillain-Barré syndrome in south-west Stockholm, 1973-91. 1. Quality of registered hospital diagnoses and incidence. Acta Neurol Scand 1995;91:109-13.

14 McLean M, Duclos P, Jacob P, et al. Incidence of GuillainBarré syndrome in Ontario and Quebec, 1983-9, using hospital services databases. Epidemiology 1994;5:443-8.

15 Hart DE, Rojas LA, Rosario JA, et al. Childhood Guillain-Barré syndrome in Paraguay, 1990-1. Ann Neurol 1994;36: 859-63.

16 Sedano MJ, Calleja J, Canga E, et al. Guillain-Barré syndrome in Cantabria, Spain. An epidemiological and clinical study. Acta Neurol Scand 1994;89:287-92.

17 Farkila M, Kinnunen E, Weckstrom P. Survey of GuillainBarré syndrome in southern Finland. Neuroepidemiology Barre syndrome

18 Winner SJ, Evans JG. Age-specific incidence of GuillainBarré syndrome in Oxfordshire. $O F \mathrm{Fed} 1990 ; 77: 1297-$ 304.

19 Kaplan JE, Poduska PJ, McIntosh GC, et al. Guillain-Barré syndrome in Larimer County, Colorado; a high-incidence area. Neurol 1985;35:581-4.

20 Beghi E, Kurland LT, Mulder DW, et al. Guillain-Barré syndrome: clinicoepidemiologic features and effects of influenza vaccine. Arch Neurol 1985;42:1053-7.

21 Koobatian TJ, Birkhead GS, Schramm MM, et al. The use of hospital discharge data for public health surveillance of Guillain-Barré syndrome. Ann Neurol 1991;30:618-21.

22 Tharakan J, Ferner RE, Hughes RAC. Plasma exchange fo Guillain-Barré syndrome. $\mathcal{F} R$ Soc Med 1989;82:458-61. 